\title{
A time domain induced polarization relaxation time spectrum inversion method based on a damping factor and residual correction
}

\author{
Liu Xiaonan, Kong Li, Zhou Kaibo and Zhang Pu* \\ School of Automation, Huazhong University of Science and Technology, Wuhan, Hubei 430074, China \\ (C) China University of Petroleum (Beijing) and Springer-Verlag Berlin Heidelberg 2014
}

\begin{abstract}
Relaxation time spectra (RTS) derived from time domain induced polarization data (TDIP) are helpful to assess oil reservoir pore structures. However, due to the sensitivity to the signal-to-noise ratio (SNR), the inversion accuracy of the traditional singular value decomposition (SVD) inversion method reduces with a decrease of SNR. In order to enhance the inversion accuracy and improve robustness of the inversion method to the SNR, an improved inversion method, based on damping factor and spectrum component residual correction, is proposed in this study. The numerical inversion results show that the oscillation of the RTS derived from the SVD method increased with a decrease of SNR, which makes it impossible to get accurate inversion components. However, the SNR has little influence on inversion components of the improved method, and the RTS has high inversion accuracy and robustness. Moreover, RTS derived from core sample data is basically in accord with the pore-size distribution curve, and the RTS derived from the actual induced polarization logging data is smooth and continuous, which indicates that the improved method is practicable.
\end{abstract}

Key words: Inversion method, damping factor, relaxation time spectrum, time domain induced polarization, spectrum component residual correction

\section{Introduction}

The phenomenon of induced polarization was discovered in 1920 (Schlumberger, 1920). Due to numerous advantages such as low cost, deep detecting depth, large signal amplitude and high SNR, the TDIP method is widely used in many fields (Corrêa et al, 2009; Titov et al, 2010; Gazoty et al, 2012; Fiandaca et al, 2012; 2013). Vinegar and Waxman showed that the polarization potential decay curve had a close relationship with the formation pore structure, and the relaxation time was proportional to the square of the pore radius (Vinegar and Waxman, 1987; 1988). Therefore, the RTS derived from the polarizability decay curve, instead of a single relaxation time value, is quite attractive in studying the characteristics of formations (Morgan and Lesmes, 1994; Paulson et al, 2000; Titov et al, 2002; Tong et al, 2005; 2006a; Tong and Tao, 2007; Wang et al, 2011). However, the raw decay data is usually corrupted by noise, restricting the inversion accuracy (Wahba, 1977). So far, although many developments have been seen in instrument design and acquisition techniques, the inversion methods commonly used still cannot gain satisfying results. Consequently, it is crucial to develop an inversion method to improve the robustness of RTS to noise.

*Corresponding author. email: zhangpu3430@hust.edu.cn

Received June 14, 2013
The essence of RTS inversion is multi-exponential fitting. There are many usages of the multi-exponential inversion method in the inversion of magnetic resonance relaxation signals (Xiao et al, 2004; 2012; Liao et al, 2007; Tan et al, 2008; Mohnke and Yaramanci, 2008), but it is seldom used in TDIP. Tong et al (2004) used the SVD method to transform induced polarization decay data into RTS for the first time, and estimated permeability of a shaly sand reservoir. Based on the regularized solution of the Fredholm integral equation, Tarasov and Titov proposed an algorithm for the inversion of relaxation time distribution from TDIP data (Tarasov and Titov, 2007). In contrast with their methods, we pay more attention to the robustness to SNR and inversion accuracy in this study.

In this study, an improved inversion method, based on a damping factor and the spectrum component residual correction non-negative constraint method, is therefore proposed to reduce the sensitivity of RTS to noise and enhance the inversion accuracy. First, we briefly describe the basic principles of TDIP. Second, we introduce the improved RTS inversion method in detail. Third, numerical inversion is used to verify the availability of the improved method and we discuss the influence of SNR on the inversion results. Finally, the practicability of improved inversion method is discussed through the inversion from the core sample and actual induced polarization (IP) logging data. 


\section{TDIP basic principle}

Induced polarization phenomenon is a feature of ionic conductive minerals under an applied electric field. As is shown in Fig. 1(a), the excess negative charges on the surface of clay particles attract cations, forming an electrical double layer with a dispersion structure. If the rock particle pore lies in the diffusion layer, the excess attracted cations will attract anions and repel cations. As is shown in Fig. 1(b), under the applied electric field, because the mobility of cations is greater than anions in the diffusion layer, a cation selective permeable membrane is formed in the pore of rock which contains clay particles. When an external electric field is applied at both ends of the pore, ion concentration gradient and polarization potential emerge. After the electric field is removed, the gradient disappears gradually with ion diffusion and the ion concentration comes back to the initial state, forming the polarization potential decay curve (Vinegar and Waxman, 1984; Jiang et al, 1999; Tarasov and Titov, 2007; Guan et al, 2010; Li et al, 2011).

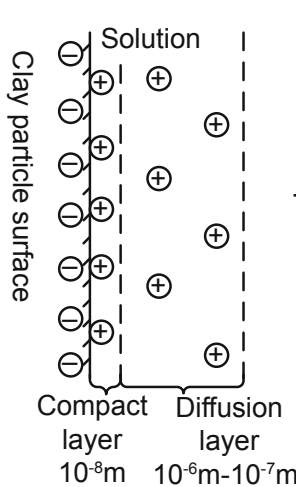

(a)

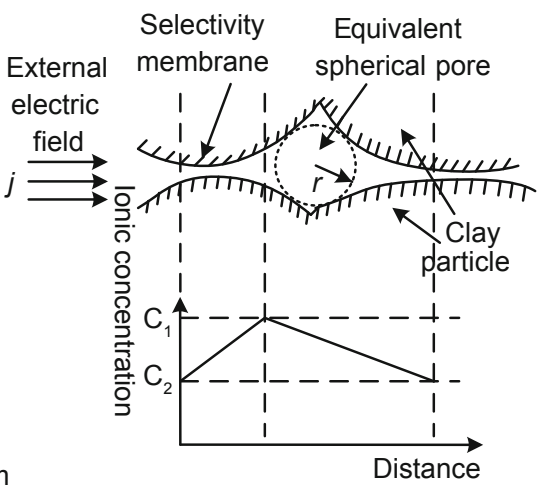

(b)
Fig. 1 Induced polarization phenomenon (a) Electric double layer with dispersion structure; (b) Effect of membrane polarization

\section{Relaxation time spectrum inversion method}

During induced polarization, both an ion concentration gradient is formed and ion diffusion takes place along the direction of the external electric field. Therefore, the relaxation diffusion distance is related to the pore diameter, and the size distribution of pore diameters can be obtained from the relaxation time. Studies show that the polarization decay potential of a single pore satisfies the single exponential decay law (Vinegar and Waxman, 1987; 1988), and the time constant $T$ is given by

$$
T=\frac{r^{2}}{D}
$$

where $D$ is the diffusion coefficient of a dielectric ion in the pore, $r$ is the radius of an equivalent spherical pore as is shown in Fig. 1(b). However, the actual polarizability decay curve represents a superposition of a series of exponential decay functions with different time constants, as is shown in Eq. (2)

$$
\eta\left(t_{i}\right)=\sum_{j=1}^{n} f_{j} \cdot \exp \left(-\frac{t_{i}}{T_{j}}\right)
$$

where $t_{i}(i=1,2, \ldots, m)$ is the measurement time point of the decay potential, $T_{j}(j=1,2, \ldots, n)$ is relaxation time constants designated in advance, and $f_{j}(j=1,2, \ldots, n)$ is spectral amplitude relating to $T_{j}$, representing the share of the $j$ th pore volume in total porosity. Then after the amplitude $f$ is obtained by multi-exponential inversion, the RTS is defined as the curve of amplitude $f$ versus relaxation time $T$. The different relaxation time constants are relevant to different pore radii, so the RTS reflects the pore size distribution.

\subsection{Constraint analysis of optimization model}

As the problem is ill-posed, the solution may be not unique or there may be no exact solution, so Eq. (2) is recast as a least square optimization problem with a non-negative constraint

$$
\min \|J \cdot f-\eta\|^{2} \quad \text { s.t. } f_{j} \geq 0
$$

where $J_{i j}=\exp \left(-\frac{t_{i}}{T_{j}}\right)$ is the coefficient matrix. To solve the optimization problem and obtain the least squares solution, the SVD method has been adopted (Tong et al, 2006b; Wang et al, 2001). However, the solution is sensitive to noise, and can easily have serious oscillation, which is inconsistent with the continuity of pore size distribution.

To eliminate the abnormality and smooth the inversion result, the damping least square (DLS) method is proposed to obtain the RTS, and the spectrum component residual correction method is used to restrict the result in this study. Based on the improved method, the optimization problem is converted into Eq. (4)

$$
\min \left(\|\eta-J f\|^{2}+\alpha^{2}\|W f\|^{2}\right) \quad \text { s.t. } f_{j} \geq 0
$$

where $\alpha^{2}$ is the damping factor applied to reduce the sensitivity to noise (Provencher, 1982), $W$ is the regularization operator and different regularization operators correspond to different model constraints.

Tong and Tao (2007) chose the unit matrix as the regularization operator to restrict the model, which means that the problem is solving the optimization problem with a minimum length model. Except for the unit matrix, there are two commonly used operators, flattest and smoothest models, in inversion of geophysics which are respectively used to restrict the first derivative and second derivative of the model. Considering that the flattest model is used to restrict the derivative of the model but does not require the specific values of the model parameters, and it does not smooth the solution like the smoothest model, then in this study the flattest model is chosen as the regularization operator

$$
W=\left[\begin{array}{ccccc}
-1 & 1 & & & \\
& -1 & 1 & & \\
& & & \ddots & \\
& & & -1 & 1
\end{array}\right]_{(n-1) \times n}
$$


Then the solution of Eq. (4) meets the following matrix form

$$
\left(J^{\mathrm{T}} J+\alpha^{2} W^{\mathrm{T}} W\right) f=J^{\mathrm{T}} \eta \quad \text { s.t. } f_{j} \geq 0
$$

Through decomposing the matrix $J$ and calculating the generalized inverse matrix, the optimization solution can be obtained.

\subsection{Non-negative constraint method}

Considering the physical significance of RTS derived from TDIP data, a non-negative constraint must be applied to eliminate the negative component of the solution. For the traditional SVD non-negative constraint method, when there is a negative component in the solution, the corresponding column of the coefficient matrix will be eliminated. Then the obtained RTS does not include the component corresponding to the column, so eliminating the negative component. However, because the dimension of the coefficient matrix is reduced, there is a lack of coefficient matrix information and the RTS is discontinuous. Moreover, the SVD of the coefficient matrix will be conducted every iteration, which requires extra computer memory and time, especially when there are many relaxation time constants designated in advance.

In order to overcome the defect of the traditional non-negative constraint method and to obtain continuous RTS, the spectrum component residual correction constraint method is proposed in this study. The procedure is as follows:

Step 1: The initial solution $f$ is solved and $\eta^{\prime}=J f$ is calculated.

Step 2: The error of polarizability $\Delta \eta=\eta-\eta^{\prime}$ is obtained.

Step 3: We get the residual $\Delta f$ using Eq. (5) by $\Delta \eta$.

Step 4: Correct spectrum components by $f^{k+1}=f^{k}+\Delta f$, where $k$ means $k$ th iteration.

Step 5: If all components of the solution satisfy $f_{j} \geq 0$, the solution is accepted. Otherwise, the negative component is set to zero, and the iteration continues until the solution is acceptable.

During the iteration, compared with the conventional SVD non-negative constraint method, the coefficient matrix $J$ is not changed. Therefore, there is no information component lost, which makes the RTS smooth and continuous. Moreover, the SVD of the coefficient matrix is conducted only once, which reduces the amount and time of the computation.

\section{Results and discussion}

\subsection{Numerical inversion results}

In order to verify the availability of the improved inversion method, we make a theoretical model with four time constants, as is shown in Eq. (6)

$$
\begin{aligned}
\eta(t)= & 18 \exp \left(-\frac{t}{7}\right)+11 \exp \left(-\frac{t}{90}\right) \\
& +15 \exp \left(-\frac{t}{900}\right)+23 \exp \left(-\frac{t}{10000}\right)
\end{aligned}
$$

The theoretical decay signal is shown in Fig. 2. The measurement time range is from $2 \mathrm{~ms}$ to $120,000 \mathrm{~ms}$. Considering the resolution and computation of the relaxation spectrum, 128 relaxation time points are logarithmically selected from the measurement time range. There is no uniform method for selecting the damping factor so far. In this study, we set the damping factor as 0.005 according to inversion results and documents (Wang et al, 2001; Tong and Tao, 2007).

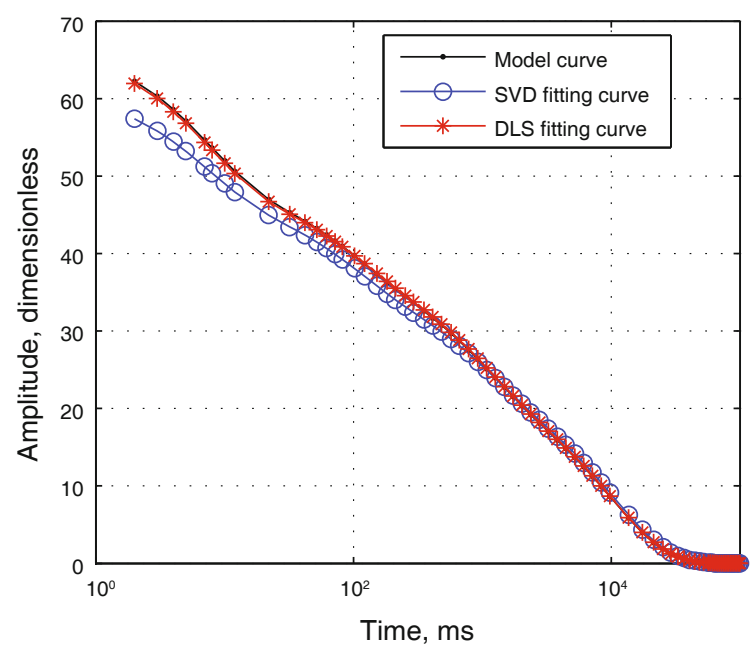

Fig. 2 Theoretical model decay curve and inversion fitting curves

As is shown in Fig. 3, the RTS derived from the SVD method has oscillation and the inversion components contain some interfering components, which are inconsistent with the theoretical model. However, because of the effect of damping factor, the RTS derived from the DLS method eliminates the oscillation, and four inversion components can be clearly distinguished.

The comparison of inversion components between the SVD and DLS methods is shown in Table 1. Because of the oscillation of the SVD method, the relaxation time points with bigger amplitude are chosen as components. As regards the DLS method, the relaxation time points of the peaks of RTS are the time of inversion components, and the amplitude of inversion components numerically equals the area encircled by the peaks. By the comparison, the SVD inversion method cannot obtain accurate time points of components. However, the DLS method has accurate time and amplitude of components. The fitting curves of SVD and DLS inversion components are shown in Fig. 2 and the residual variance is 1.429 and 0.126 respectively, which indicates that the accuracy of the improved method is significantly superior to the SVD inversion method.

\subsection{Influence of SNR on inversion results}

There is noise in the actual measured induced polarization decay signal, and the SNR has a great influence on the inversion accuracy. Therefore, in order to study the influence of SNR on inversion results, Gauss white noise designated 50 $\mathrm{dB}$ and $20 \mathrm{~dB}$ SNR is superimposed on the theoretical signal. Then the RTS derived from the SVD and DLS methods are shown in Fig. 4 and Fig. 5 respectively. The damping factors 



Fig. 3 RTS derived from SVD and DLS methods

of the DLS method, selected in the same way as described in Section 4.1 , are 0.5 and 2 respectively in $50 \mathrm{~dB}$ and $20 \mathrm{~dB}$. The inversion components are shown in Table 2, and the time and amplitude of the inversion components are determined by the same way as Table 1 .

According to the inversion results, the residual variance of fitting curves derived from SVD inversion components in $50 \mathrm{~dB}$ and $20 \mathrm{~dB}$ SNR is 3.149 and 7.140 respectively, while the DLS method is 0.547 and 2.065. The inversion results show that the inversion accuracy of both methods decreases with the reduction in the SNR. But the inversion accuracy of the DLS is superior to that of the SVD and the SNR has little influence on the inversion components of DLS, which means the DLS method has good robustness to SNR.

\subsection{Application and discussion}

According to the RTS theory, the RTS reflects the pore size distribution, which has practical significance for petroleum exploration and production. In order to verify the
Table 1 Comparison of inversion components

\begin{tabular}{|c|c|c|c|c|c|c|}
\hline \multicolumn{7}{|c|}{ Theoretical model components } \\
\hline$T, \mathrm{~ms}$ & 7 & \multicolumn{2}{|c|}{90} & 900 & \multicolumn{2}{|c|}{10000} \\
\hline Amplitude & 18 & \multicolumn{2}{|l|}{11} & 15 & \multicolumn{2}{|c|}{23} \\
\hline \multicolumn{7}{|c|}{ Inversion components from SVD } \\
\hline$T, \mathrm{~ms}$ & 6.707 & 7.973 & 89.674 & 778.2 & 925.1 & 10404 \\
\hline Amplitude & 14.154 & 4.535 & 11.116 & 2.897 & 12.729 & 23.326 \\
\hline \multicolumn{7}{|c|}{ Inversion components from DLS } \\
\hline$T, \mathrm{~ms}$ & 7.010 & & 89.674 & & 886.77 & 9973.7 \\
\hline Amplitude & 18.129 & & 10.330 & & 15.090 & 23.166 \\
\hline
\end{tabular}

practicability of the improved inversion method, data from core samples and actual logging are used for inversion of RTS.

Three $\mathrm{NaCl}$ solutions with different salinities $(3,000$ $\mathrm{mol} / \mathrm{L}, 7,000 \mathrm{~mol} / \mathrm{L}$ and $11,000 \mathrm{~mol} / \mathrm{L}$ ) are applied to one oilfield core sample. The porosity of the core sample is $19.2 \%$ and the permeability is $3.57 \mathrm{mD}$. The polarizability
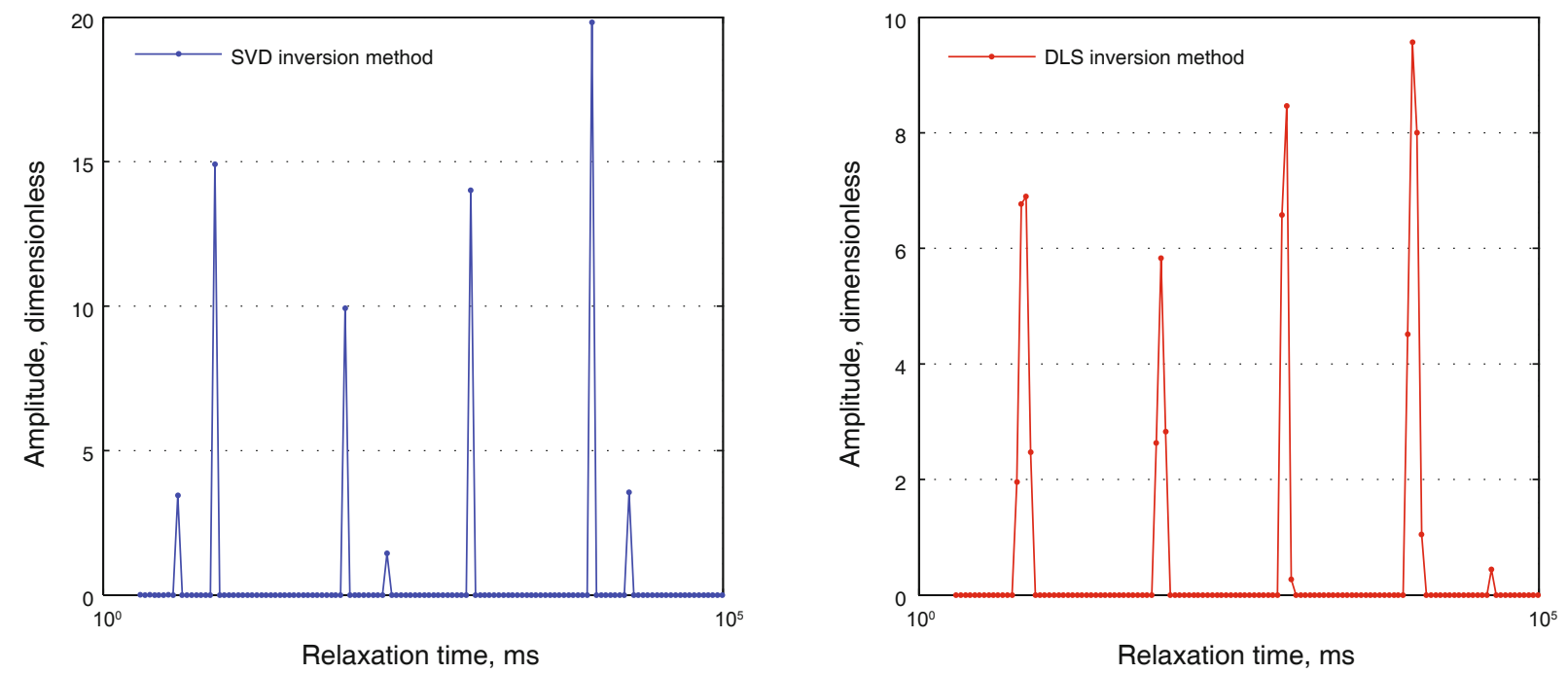

Fig. $4 \mathrm{SNR}=50 \mathrm{~dB}$, RTS derived from the SVD and DLS methods 

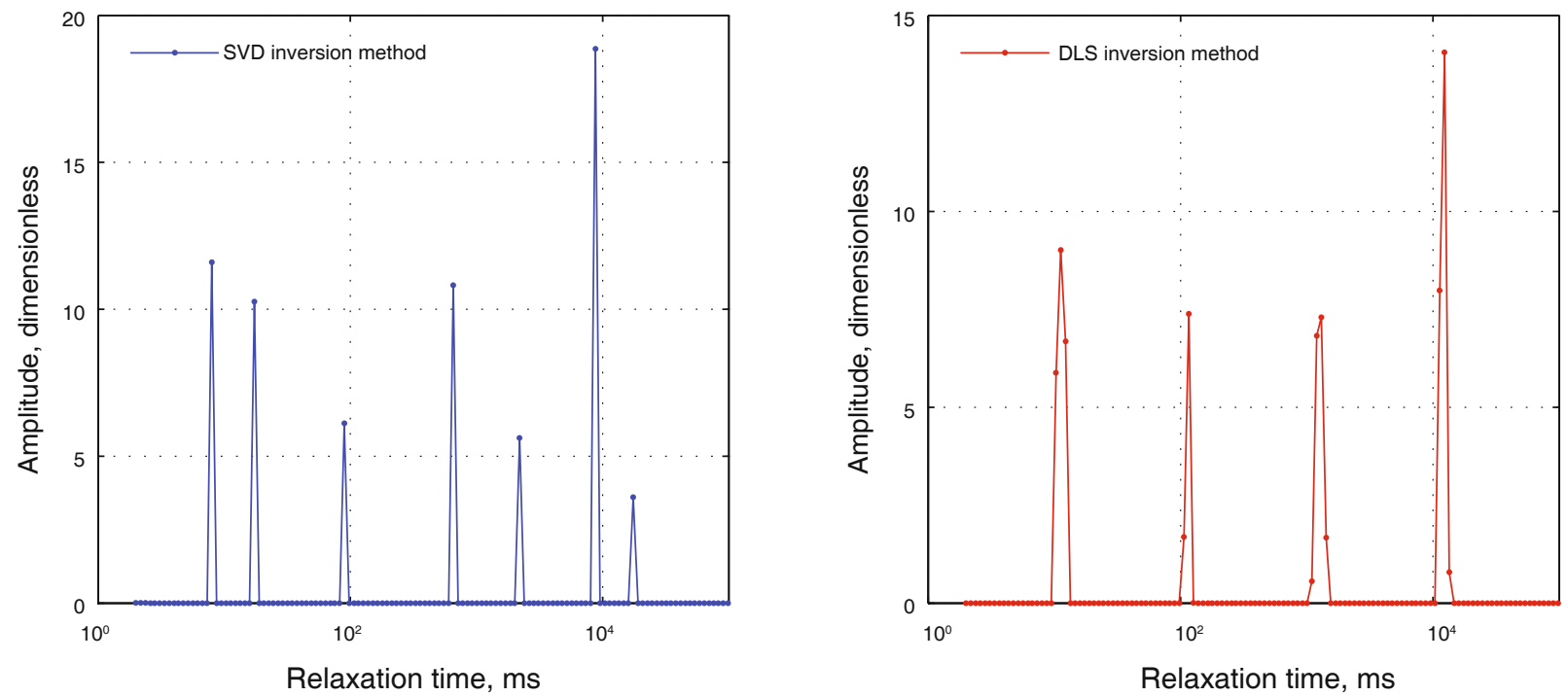

Fig. $5 \mathrm{SNR}=20 \mathrm{~dB}$, RTS derived from SVD and DLS methods

Table 2 Comparison of inversion components

\begin{tabular}{|c|c|c|c|c|c|c|}
\hline \multicolumn{7}{|c|}{ Theoretical model components } \\
\hline$T, \mathrm{~ms}$ & 7 & \multicolumn{2}{|c|}{90} & \multicolumn{2}{|c|}{900} & 10000 \\
\hline Amplitude & 18 & \multicolumn{2}{|c|}{11} & \multicolumn{2}{|c|}{15} & 23 \\
\hline \multicolumn{7}{|c|}{$\mathrm{SNR}=50 \mathrm{~dB}$, inversion components from SVD } \\
\hline$T, \mathrm{~ms}$ & 3.993 & 7.973 & 89.67 & 925.1 & 8753 & 17480 \\
\hline Amplitude & 3.449 & 14.91 & 9.935 & 14.01 & 19.82 & 3.555 \\
\hline \multicolumn{7}{|c|}{$\mathrm{SNR}=50 \mathrm{~dB}$, inversion components from DLS } \\
\hline$T, \mathrm{~ms}$ & 7.313 & \multicolumn{2}{|c|}{89.67} & \multicolumn{2}{|c|}{925.1} & 9543 \\
\hline Amplitude & 18.092 & \multicolumn{2}{|c|}{11.285} & \multicolumn{2}{|c|}{15.310} & 23.128 \\
\hline \multicolumn{7}{|c|}{$\mathrm{SNR}=20 \mathrm{~dB}$, inversion components from SVD } \\
\hline$T, \mathrm{~ms}$ & 7.973 & 17.36 & 89.67 & 654.7 & 2196 & 8753 \\
\hline Amplitude & 11.60 & 10.26 & 6.117 & 10.82 & 5.627 & 18.87 \\
\hline \multicolumn{7}{|c|}{$\mathrm{SNR}=20 \mathrm{~dB}$, inversion components from DLS } \\
\hline$T, \mathrm{~ms}$ & 11.27 & & 5.2 & & & 12370 \\
\hline Amplitude & 21.53 & & 72 & & & 22.83 \\
\hline
\end{tabular}

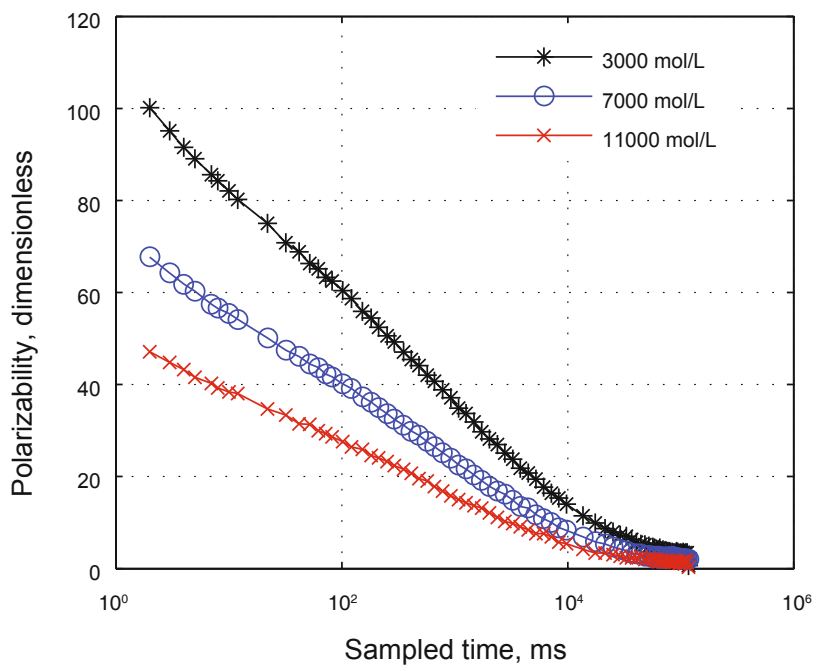

(a) decay curve and the normalization polarizability decay curve of the core sample are shown in Fig. 6. Fig. 6(a) shows that because the resistivity of the sample decreases with an increase of salinity, the polarization potential becomes small and the polarizability decay signal is reduced. However, normalization reveals that the decay signal is only related to the pore structure of the formation, and has no relationship with the value of polarizability. Therefore, the salinity has almost no influence on the normalization polarizability decay signal, as is shown in Fig. 6(b).

In this study, according to the improved inversion method, the RTS are obtained from the normalization decay signal at different salinities, and the RTS can be converted into a relaxation radius spectrum by Eq. (1) (knowing that the diffusion coefficient $D$ of $\mathrm{NaCl}$ ions in solution is $1.5 \times 10^{-9} \mathrm{~m}^{2} / \mathrm{s}$ at room temperature), shown in Fig. 7. The actual pore-size distribution curve in Fig. 7 is from the pore feature image of casting thin sections (shown in Fig. 8). The comparison between relaxation radius spectra and pore-size distribution shows that the relaxation radius spectra are basically consistent with the pore size distribution curve,



(b)

Fig. 6 Core sample polarizability decay curve with different salinities. (a) Polarizability decay curve, (b) Normalized polarizability decay curve 


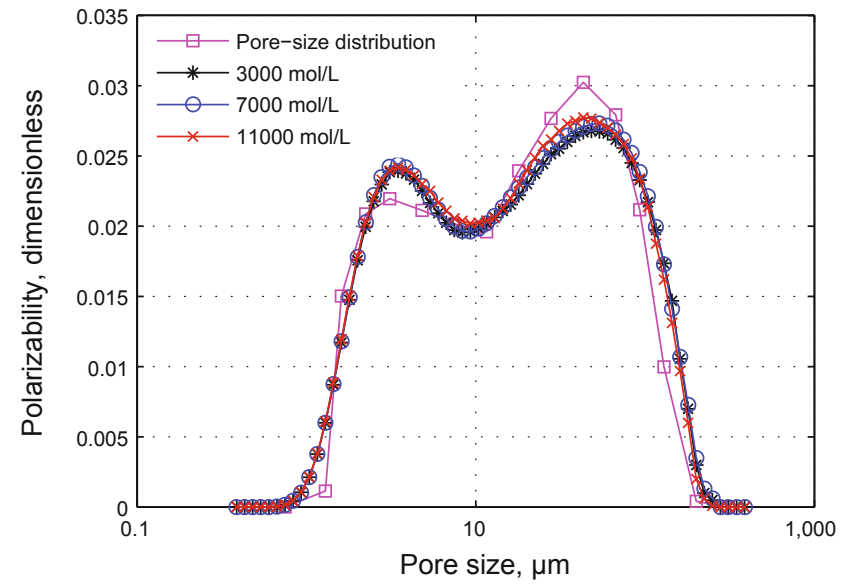

Fig. 7 Pore size distribution and IP relaxation radius spectra with different salinities

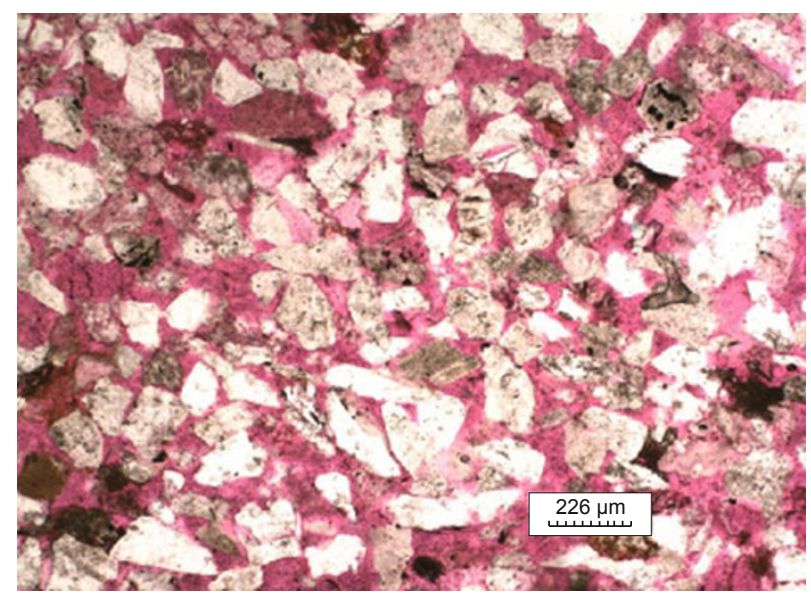

Fig. 8 Pore feature image of casting thin sections

and the pore diameter is $1-200 \mu \mathrm{m}$.

In addition, Fig. 9(a) is actual induced polarization logging data, and (b) is RTS inversion result, which indicates that the RTS derived from the improved inversion method is smooth and continuous, and the improved method is practicable.

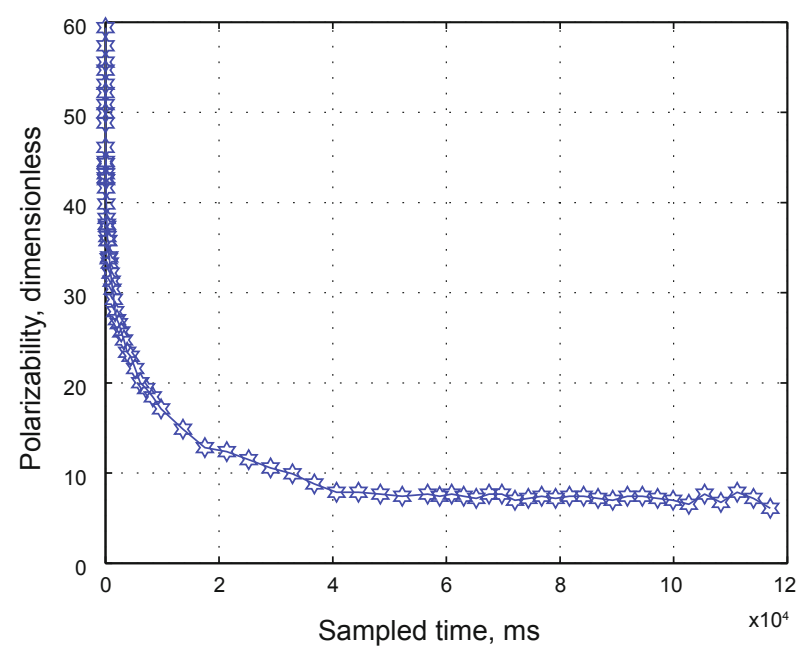

(a)

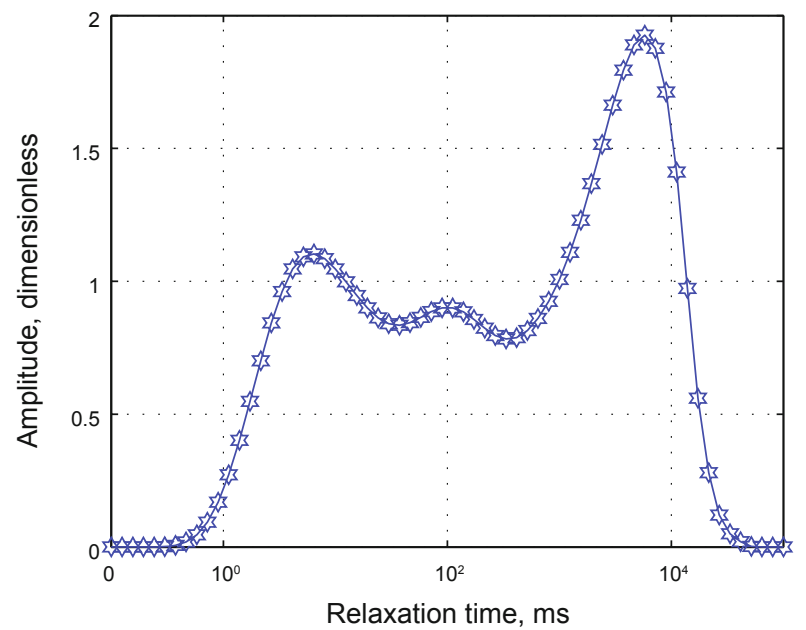

(b)

Fig. 9 IP logging curve and RTS derived from inversion (a) IP logging polarizability decay curve, (b) RTS derived from inversion

\section{Conclusions}

For the conventional SVD inversion method, the RTS has oscillation, and gets worse following any decrease in the SNR. In this study, under the effect of the damping factor and the spectrum component residual correction iteration method, the RTS derived from the improved method is smooth and continuous, and is little affected by the SNR. According to the analysis results of numerical inversion, for the SVD method, the SNR has a great influence on the RTS, so we cannot get correct inversion components. But the improved method has good inversion components in infinite, $50 \mathrm{~dB}$ and $20 \mathrm{~dB}$ SNR, which indicates that the improved method effectively reduces the sensitivity of RTS to the SNR and ensures the robustness. Furthermore, the inversion results of core samples show that the relaxation radius spectra are basically consistent with the actual pore size distribution, and the RTS derived from actual logging data is smooth and continuous, which indicates that the improved inversion method proposed in this study is practicable.

\section{Acknowledgements}

This work was partially supported by a project from the Youth Science Foundation of the National Natural Science Foundation of China (11104089).

\section{References}

Corrêa Alegria F, Martinho E and Almeida F. Measuring soil contamination with the time domain induced polarization method using LabVIEW. Measurement. 2009. 42(7): 1082-1091

Fiandaca G, Auken E, Christiansen A V, et al. Time-domain-induced polarization: Full-decay forward modeling and 1D laterally constrained inversion of Cole-Cole parameters. Geophysics. 2012. 77(3): E213-E225

Fiandaca G, Ramm J, Binley A, et al. Resolving spectral information from time domain induced polarization data through 2-D inversion. Geophysical Journal International. 2013. 192(2): 631-646 
Gazoty A, Fiandaca G, Pedersen J, et al. Application of time domain induced polarization to the mapping of lithotypes in a landfill site. Hydrology and Earth System Sciences. 2012. 16(6): 1793-1804

Guan J T, Wang Q, Fan Y H, et al. Study on the mechanisms of electrochemical logging response in shaly sandstone based on capillary model. Chinese Journal of Geophysics. 2010. 53(1): 214223 (in Chinese)

Jiang E C, Wang J N, Sun B D, et al. New advances in theoretical study and practical application of electrochemical logging. Well Logging Technology. 1999. 23(5): 327-333 (in Chinese)

Li Y, Lin P R, Xiao Y, et al. Induced polarization effect on frequencydomain electromagnetic sounding with electric dipole source. Chinese Journal of Geophysics. 2011. 54(7): 1935-1944 (in Chinese)

Liao G Z, Xiao L Z, Xie R H, et al. Influence factors of multiexponential inversion of NMR relaxation measurement in porous media. Chinese Journal of Geophysics. 2007. 50(3): 932-938 (in Chinese)

Mohnke $\mathrm{O}$ and Yaramanci U. Pore size distributions and hydraulic conductivities of rocks derived from magnetic resonance sounding relaxation data using multi-exponential decay time inversion. Journal of Applied Geophysics. 2008. 66(3): 73-81

Morgan F D and Lesmes D P. Inversion for dielectric relaxation spectra. Journal of Chemical Physics. 1994. 100(1): 671-681

Paulson K S, Jouravleva S and McLeod C N. Dielectric relaxation time spectroscopy. Biomedical Engineering, IEEE Transactions on. 2000. 47(11): 1510-1517

Provencher S W. A constrained regularization method for inverting data represented by linear algebraic or integral equations. Computer Physics Communications. 1982. 27(3): 213-227

Schlumberger C. Etude sur la prospection electrique du sous-sol. Gauthier-Villars. 1920

Tan M J, Shi Y L, Zhao W J, et al. A joint inversion method for NMR dual-TW logging data and fluid typing. Chinese Journal of Geophysics. 2008. 51(5): 1100-1109 (in Chinese)

Tarasov A and Titov K. Relaxation time distribution from time domain induced polarization measurements. Geophysical Journal International. 2007. 170(1): 31-43

Titov K, Komarov V, Tarasov V, et al. Theoretical and experimental study of time domain-induced polarization in water-saturated sands. Journal of Applied Geophysics. 2002. 50(4): 417-433

Titov K, Tarasov A, Ilyin Y, et al. Relationships between induced polarization relaxation time and hydraulic properties of sandstone. Geophysical Journal International. 2010. 180(3): 1095-1106

Tong M, Li L, Wang W, et al. Estimation of pore size distribution and permeability of shaly sands from induced polarization time spectra. Chinese Journal of Geophysics. 2005. 48(3): 785-791 (in Chinese)

Tong M, Li L, Wang W, et al. A time-domain induced-polarization method for estimating permeability in a shaly sand reservoir. Geophysical Prospecting. 2006a. 54(5): 623-631

Tong M, Li L, Wang W, et al. Determining capillary-pressure curve, pore-size distribution, and permeability from induced polarization of shaley sand. Geophysics. 2006b. 71(3): N33-N40

Tong $\mathrm{M}$ and Tao $\mathrm{H}$. Experimental study of induced polarization relaxation time spectra of shaly sands. Journal of Petroleum Science and Engineering. 2007. 59(3): 239-249

Tong M, Wang W, Li L, et al. Estimation of permeability of shaly sand reservoir from induced polarization relaxation time spectra. Journal of Petroleum Science and Engineering. 2004. 45(1-2): 1-10

Vinegar H J and Waxman M H. Induced polarization of shaly sands. Geophysics. 1984. 49(8): 1267-1287

Vinegar H J and Waxman M H. In-situ method for determining pore size distribution, capillary pressure and permeability. U.S. Patent 4,644,283. 1987-2-17

Vinegar H J and Waxman M H. In-situ induced polarization method for determining formation permeability. U.S. Patent 4,743,854. 1988-5 $-10$

Wahba G. Practical approximate solutions to linear operator equations when the data are noisy. SIAM Journal on Numerical Analysis. 1977. 14(4): 651-667

Wang W, Li P and Ye C. Multi-exponential inversions of nuclear magnetic resonance relaxation signal. Science in China Series A: Mathematics. 2001. 44(11): 1477-1484

Wang W N, Yu Q F, Tong M S, et al. Electrode configuration design of induced polarization potential decay spectrum logging. Progress in Geophysics. 2011. 26(1): 371-375 (in Chinese)

Xiao L, Wang $\mathrm{Z}$ and Liu T. Application of multi-exponential inversion method to NMR measurements. Petroleum Science. 2004. 1(1): 1922

Xiao L Z, Zhang H R, Liao G Z, et al. Inversion of NMR relaxation in porous media based on Backus-Gilbert theory. Chinese Journal of Geophysics. 2012. 55(11): 3821-3828 (in Chinese)

(Edited by Hao Jie) 